\title{
'I do not think there is a "magic bullet" solution'
}

Kate Quinlan seeks views on the sugar tax and its potential impact on oral health.

$\int$ n ntroduction

In March 2016, the UK Chancellor would be a levy on sugar sweetened beverages from 2018, due to the obesity crisis and related health conditions such as diabetes and dental caries. This sugared soft drinks levy would have a banding structure: less than $5 \mathrm{~g}$ per 100 $\mathrm{ml}$, no tax; 5-8 g per $100 \mathrm{ml}$, basic level tax; and more than $8 \mathrm{~g}$ per $100 \mathrm{ml}$, higher level tax.

As soon as the announcement was made, drinks manufacturers started altering their formulations so that some drinks contained less sugar.

When the levy came into force on 6 April 2018 it was expected to raise around $£ 520$ million a year, to be spent on increasing sport funding in primary schools. No funds were specifically pledged towards improving oral health education or action to help reduce the impact of sugar on teeth. In addition, the levy on sugar sweetened beverages was not extended to pure fruit juices, milk-based drinks and multi-packs.

This summer Westminster were considering extending the soft drinks levy to 'sugary milk drinks', and Public Health England has urged firms that make milkshakes to voluntarily implement a $20 \%$ sugar reduction by mid-2021 [the food industry has also been called on to reduce sugar in their most popular food products by $20 \%$ by 2020 ]. However, in June Boris Johnson hinted that he might scrap plans for a 'milkshake tax' that would 'clobber those who can least afford it'. Despite this, the Green Paper published on 22 July, hours before Boris Johnson was elected Tory leader, suggests that a tax on sugary milk drinks may still be introduced. ${ }^{1}$

According to the British Dental Association (BDA) in April this year, no specific revenue from the sugar levy has been earmarked to combat dental caries.

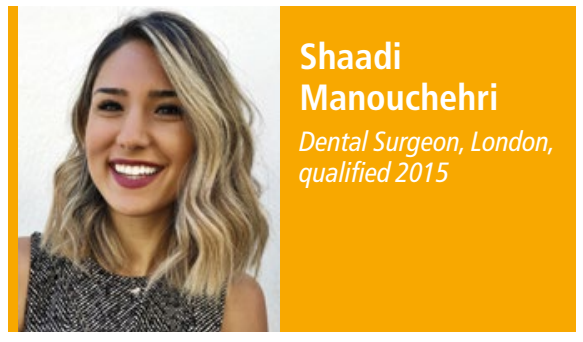

Were you/are you in favour of the levy on sugar sweetened beverages (SSB) (or 'sugar tax') being introduced? The levy on SSBs could be a great initiative in attempting to combat the negative effects of excessive sugar consumption on one's overall health as well as in reducing the occurrence of dental caries amongst the population.

Do you think that this will reduce levels of dental caries among your patients, in the short or long term? There is great potential for the levy on SSBs to contribute to the reduction of the level of dental caries amongst the population and this is likely to be in the long term rather than the short term. The addition of this tax may not only potentially deter the consumer but has already been shown to encourage manufacturers to reduce the sugar content of their products. in primary schools and there has been no mention of allocating some of these funds to improving oral health education or other such initiatives to improve oral health.

Given that dental caries-related extractions under general anaesthetic remain the number one cause for children in the UK being admitted to hospital; one would expect there to be greater emphasis on this. Furthermore, dental caries amongst children has been linked to pain, distress, reduced quality of life, sleepless nights for patients and parents and also a greater number of days missed from school or work. Therefore, it is important to highlight the link between frequent sugar consumption and dental health as well as overall health.

Have your patients raised the topic of the sugar tax/sugar in fizzy drinks, or commented on their consumption of these drinks with you? There has been very little comment on the sugar tax, however diet advice is being routinely provided to patients who report a high intake of sugary/fizzy drinks.

Has your dental practice developed oral health educational initiatives regarding the levels of sugar in food and drink? Verbal and written diet advice is routinely provided to all patients and we have introduced

\section{'According to the BDA in April this year, no specific revenue from the sugar levy has been earmarked to combat dental caries.'}

However, it is important to note that the tax alone may not be sufficient and will almost certainly need to be supplemented by oral health education projects to raise awareness.

There is great emphasis in this campaign on the overall health impact of these changes but not the anticipated effect on dental health. It is important to note that the expected funds raised are to be spent on sports funding visual aids in the practice in order to raise awareness of the sugar content of certain food and beverages. This has been particularly well received by our patients, especially parents of paediatric patients, and we have been able to observe a reduction in caries rate amongst these patients.

Do you think further taxes should be placed on other sugar-containing foods 
and drinks? Perhaps; it is difficult to anticipate the effect of further taxes alone. I believe we should also focus on oral health education initiatives to raise awareness of the sugar content in foods, especially hidden sugars in 'healthy foods' such as dried fruits and fruit juices. As there is now more emphasis on maintaining a healthy diet, we must ensure the public are aware that naturally occurring sugars such as those in dried fruits can also contribute to tooth decay.

Campaigns to increase awareness and educate the public can prove successful. These campaigns would ideally include information, evidence based advice and helpful tips on realistic changes that the public can introduce to their daily routines in order to improve their dental and therefore overall health.

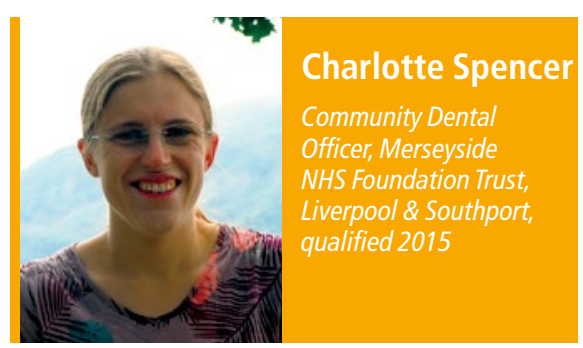

Were you/are you in favour of the levy on sugar sweetened beverages being introduced? I think that the sugar tax is a great idea to encourage people to make healthier choices. When shopping it is healthy foods such as fruit and veg that are often more expensive than processed foods which are generally high in both sugar and salt. In my opinion introducing a taxation incentive to promote healthier foods cannot be a bad thing if done so in the right way.

Do you think that this will reduce levels of dental caries among your patients, in the short or long term? Changing attitudes and behaviours takes time. There is never a 'quick fix'. Many of the patients who have been referred to our service for dental extractions, often under general anaesthetic, have been given preventative advice previously. In many instances we find that the same dental issues have happened with siblings. There is clearly a wider issue and no one incentive will itself reduce the levels of dental caries. Education is crucial.

Have your patients raised the topic of the sugar tax/sugar in fizzy drinks, or commented on their consumption of these drinks with you? None of my patients or their parents/carers have mentioned the sugar tax.

I did once have a teenage patient comment on the ban on energy drinks to under 16s.

As part of our assessment patients are always asked about their diet. One question I ask each time is what drinks they have. Parents are thinking about their buying habits. They try to do the right thing by buying 'no added sugar' cordial or fruit juice as opposed to fizzy drinks or 'normal' cordial. I explain that these drinks are still high in sugar, they just haven't had any sugar added by the manufacturer.

Water and milk are always my recommendation. Sugary drinks do not have to be banned completely but they must be thought of as a treat and ideally had with a meal.

Has your place of work developed oral health educational initiatives regarding the levels of sugar in food and drink? Within our clinic we are very fortunate to have several nurses trained as Dental Health Educators. We routinely offer these appointments to patients referred to us by their GDPs for carious teeth. The feedback from these appointments is that parents find them very helpful due to the advice being tailored to their child.

Do members of your dental team conduct oral health education outside the dental practice/in the community? Merseycare have a dental promotion team that work out in the community visiting and sharing knowledge at children's centres, nursing homes, health events and to vulnerable groups. The team give information to managers and staff as well as to parents and carers. Both groups are equally as important if we are to achieve change.

The promotion team support projects from Public Health England such as 'Starting Well'. This is a commissioned approach to improve access to preventive oral healthcare for $0-2$ year-olds. This national programme aims to increase dental access and attendance for children aged 0-2 years for their first dental check-up, support for oral health behaviour change, brushing teeth twice daily with a fluoride toothpaste and having a healthy diet.

Do you think further taxes should be placed on other sugar-containing foods and drinks? I personally think that the sugar tax should be extended to other food and drink groups including pure fruit juices. Added sugar is not the only 'evil'. Ultimately, education around a healthy diet needs to be extended beyond just fatty foods and provide a wholehearted approach to improving diets.

I also think that it would be a fantastic use of resources if the revenue from the sugar tax could be used to develop a similar programme to the 'Childsmile' incentive across the UK as in Scotland.

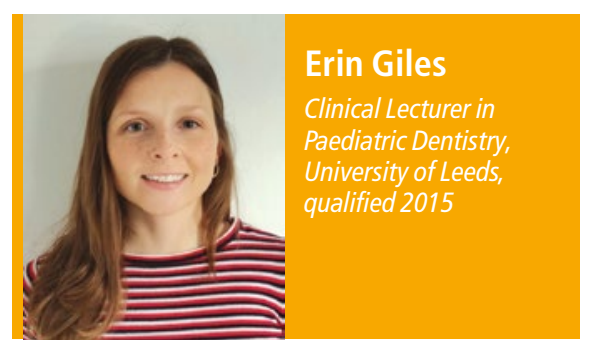

Were you in favour of the levy on sugar sweetened beverages being introduced? I was in favour of the levy. Increased taxation on sugary drinks in other countries has been shown to decrease their consumption, albeit mostly in higher socioeconomic groups. A health strategy that hits the headlines can also be of benefit beyond the fiscal technicalities, putting it higher in the public agenda, in the same way that restrictions and taxes imposed on tobacco have contributed to the incremental de-normalisation of smoking.

Do you think that this will reduce levels of dental caries among your patients, in the short or long term? The effect of the 'sugar tax' on caries levels in my patients was always going to be difficult to predict. Caries has a socioeconomic-related distribution - if the greatest decrease occurs in the lower risk groups, it will not have the biggest impact on the patients I see with diet-related dental disease. I can't say I have observed an anecdotal reduction in the consumption of sugary beverages in the high-risk caries children I treat. But it is encouraging to see that many well-known drinks manufacturers have altered their recipes. Patients are potentially consuming less sugar without needing to navigate the difficulties of changing behaviour. There will hopefully be a trickle-down benefit, but any improvement will certainly take time.

Since its enforcement 18 months ago, no parent has approached me to discuss the sugar tax. I suspect this reflects its apparent primary focus on tackling obesity, rather than dental health.

Have you been involved in oral health educational initiatives? Advances in behaviour change psychology have allowed us to make improvements in the way we give preventative advice. Through my work at the university, I have been part of research projects that have designed two novel oral health interventions: one of which is delivered by dental teams 
14 to the parents of young children (Strong

Teeth); and another given by health visitors to parents of children aged 9-12 months (HABIT). Initial early-phase studies to assess the impact of these interventions on oral health behaviours have been positive, showing an increase in compliance with $\mathrm{DBOH}$ toolkit guidelines. Any national government agenda can only be successful if there are midstream and downstream projects supporting it.

Have members of your team/colleagues been involved in oral health education or training? A dental therapist, alongside the paediatric research team, has created an excellent educational resource to develop foundation trainees' confidence and skills in undertaking behaviour change conversations. The workshop forms part of their mandatory study day programme in Yorkshire and Humber. Training all members of the dental team that give oral health education is extremely important - it's easy to fall into the trap of thinking that just because you know the guidelines, you give great preventative advice! Developing structured conversations and recognising resistance to change can be tricky but is essential to productive health promotion. These resources will soon be available to all dental teams on e-Learning for Healthcare (e-lfh.org.uk).

Do you think further taxes should be placed on other sugar-containing foods and drinks? It could go further. A large proportion of children's and lunchbox drinks are not subject to increased taxation because they fall under the category of fruit juice - smoothies are often cited as beverages with some of the highest sugar content. While the levy doesn't extend to all sugary drinks, there will be no incentive for these manufacturers to reformulate them. However, this would need to be carefully managed for fruit and milk-based products that may have other health benefits.

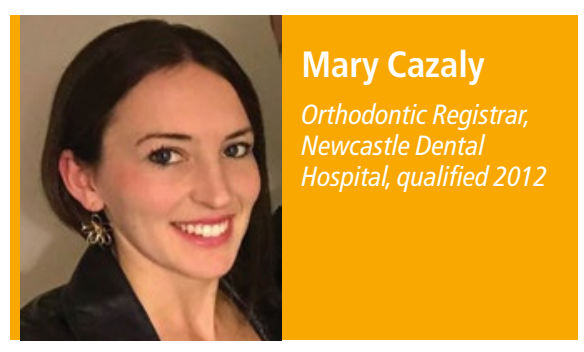

Were you in favour of the levy on sugar sweetened beverages being introduced? Yes, definitely. The government should be doing more to combat obesity and dental caries. Tooth decay is now an epidemic amongst children in the UK and is one of the main reasons for children undergoing a general anaesthetic, which still carries risks even with advances in medicine.

Do you think that this will reduce levels of dental caries among your patients? This is the hope, that the effect of the sugar levy will be more of a long term solution, although I feel this needs to be across all high sugar drinks and foods. I think it is unlikely to have much of an effect in the short term. The concern is there are other drinks which also contain high amounts of sugar. I still notice many offers in supermarkets and bargain food shops involving high sugar drinks and foods. There are often offers on confectionery including sweets and these are still sold near the till; this needs to be changed.

Despite working in an Orthodontic Department which has close links to the Paediatric Dentistry Department I have not had a single patient mention the topic of the sugar tax! Although I do feel a lot of patients and parents are now more aware of the sugar content of fizzy drinks/milkshakes, it is a frequent discussion about the content of sugar in fruit juices and other juices which are promoted as the 'healthy alternative'. I do not feel the sugar tax has influenced my patients in what they buy, as it does not include all drinks and foods containing high sugar.

Has your workplace developed oral health initiatives regarding the levels of sugar in food and drink? There is a chart displayed in the department which is a fantastic visual representative of the amount of sugar in a varied range of drinks, to highlight just how much is contained. Dietary analysis is also an important aspect of the caries prevention process. There is a drive for preventative care and general dentists are advised to follow the guidelines from the recent Delivering better oral health document. The Paediatric Dentistry Department also promotes the DCbyl, which is to promote babies having a dental check by age one.

The hospital mostly deals with the many incoming referrals. However, there are dental undergraduates and training hygienists and therapists who engage in delivering oral health education in schools and community dental practices. This includes both oral hygiene instruction and dietary advice.

Do you think further taxes should be placed on other sugar-containing foods and drinks? Yes I do feel high sugar-containing drinks and high sugar foods should be more heavily taxed. In particular, to hit the sale of confectionery and sweets in supermarkets and bargain shops. My only concern however would be that the smoothies and pure fruit juices are likely to have a health benefit in other ways, but there should certainly be a warning regarding the amount of sugar these drinks contain and still a drive to reduce the amount of sugar in these drinks.

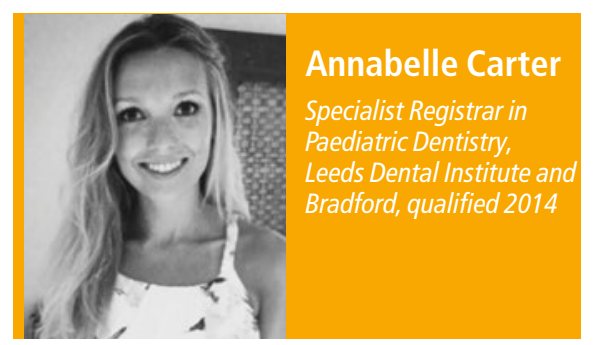

Were you in favour of the levy on sugar sweetened beverages being introduced? Yes I was. With the number of dental extraction general anaesthetics I have performed over my short career so far, I was keen to see some action that may help reduce the number of children requiring multiple teeth extractions.

I hoped the introduction of the headline-grabbing 'sugar tax' would increase awareness about the amount of sugar in certain drinks, encourage the drinks industry to reduce the amount of sugar in their drinks, and help initiate the conversations about sugars in our diet amongst our patients.

Did you think that this would reduce levels of dental caries among your patients? Short term I have not seen a reduction in the amount of dental caries, however I do see a very skewed section of the population in the dental hospital and community dental service.

I am still hopeful (and maybe too optimistic!) that it will reduce the level of dental caries among patients in the long term.

I am not as sure if it will have an impact on dental caries amongst my patients without further government policies and intervention. I am still seeing a lot of adolescents in particular with high caries levels from drinking sugary energy drinks; they have not lost their appeal among this population. I also have many patients who drink sugar-laden milkshakes - with the view that it contains milk, so 'it mustn't be that bad'. I also still have to regularly discuss the difference of no added sugar juices and sugar free juices - a conversation I am sure many will relate to. 
$4 \quad$ I am hoping in the future we may see some research that shows us some significant change in the amount of dental caries, and the impact the sugar tax has had on this.

Have your patients raised the topic of the sugar tax/sugar in fizzy drinks, or commented on their consumption of these drinks with you? When the tax was initially introduced and was headline news, it did help to initiate a conversation within the dental setting amongst my patients and their parents. This was a great starting point to discuss dietary advice in relation to dental disease and healthy living.

In my experience, the novelty of the sugar tax has waned, it has become 'old news', and the extra cost is not significant enough to make a noticeable difference to their shopping bill. If they now raise the topic of the amount of sugar in fizzy drinks, it is usually in response to poster displays they have seen in the waiting room.

Has your dental service developed oral health educational initiatives regarding the levels of sugar in food and drink? Yes, as I work across two dental services and there are some initiatives, besides the routine diet advice. In Bradford, we have a dedicated 'preventative dental unit', where an oral health educator (usually a nurse or therapist) does a full dietary analysis based on a patient's four-day diet diary they are asked to complete, or if they forget this, based on a 24-hour diet recall.

We also have pictures of a variety of

\section{Note - Sugar reduction report}

Since this perspectives feature was planned, Public Health England has published its second-year report on progress made by the food industry to voluntarily reduce sugar in everyday foods. ${ }^{4}$

Alongside looking at the reduction in sugar content in foods, the report looks at progress made under the Soft Drinks Industry Levy (SDIL). The data shows:

- A $28.8 \%$ sugar reduction per $100 \mathrm{ml}$ in retailer own brand and manufacturer branded products and a $27.2 \%$ reduction per $100 \mathrm{ml}$ for drinks consumed out of home

- There was a consumer shift towards zero or lower sugar products, with sugar purchased from soft drinks decreasing in all socio-economic groups

- 30,133 tonnes of sugar were removed without reducing soft drink sales, resulting in around 37.5 billion fewer kilocalories sold in sugary drinks each year.

However, some businesses and categories have made little or no progress so far towards reducing the sugar content in their food products by $20 \%{ }^{4}$

drinks would also help increase awareness of the amount of sugars in processed foods and 'hidden sugars', as well as hopefully putting more pressure on the food industry to reduce the sugar content in foods. This happened with many companies prior to the 2018 sugar tax, where the vast majority reduced the sugar in their drinks to below 5 grams.

I would also support this if there was transparency about where the money from the tax is going, and data that shows that the income from this tax goes towards funding schemes and health care providers that help sugar related disease and illnesses, and a proportion towards funding NHS dental services.

Would you like to share your views on what government should be doing instead/ as well, to combat obesity/diabetes/dental caries? I do not think there is a 'magic bullet' solution to combat obesity, diabetes or dental

\section{'We need to make the healthy choices easier and more affordable than the unhealthy ones, as this balance is the other way round at the moment.'}

drinks, and next to these pots containing the amount of sugar in grams, in the waiting room and in the prevention clinics. I think this helps people actually visualise how much sugar they are unknowingly consuming.

Do you think further taxes should be placed on other sugar-containing foods and drinks? Yes I do. I was shocked to discover that the percentage of sugar in chocolate bars has increased since 1992 by an average of $23 \% .^{2}$ This I am sure is not an isolated incidence in the food industry, as adding sugar to processed food can make it more appealing and better tasting. I would hope extending the sugar tax to other food and caries, and instead there should be a number of approaches to help make a difference.

As well as sugar tax, I feel there should be a collaborative approach to healthy eating and lifestyle for example, more communication and shared messages within schools, health care professionals such as health visitors, and organisations such as Diabetes UK.

I think there should be restrictions (or a ban) on supermarkets/shops for promotions on sugary, unhealthy or processed foods, to try and prevent unhealthy foods being the cheap option, and allow healthy foods to be the cheaper, more accessible and affordable option. We need to make the healthy choices easier and more affordable than the unhealthy ones, as this balance is the other way round at present. I think the government should consider curbing or preventing the amount of advertising and marketing the food industry has towards unhealthy foods, as the industry spent $£ 256$ million on advertising and marketing unhealthy foods in 2014, compared to the 'Change4Life' healthy living campaign, which the government spent $\mathfrak{£} 3.9$ million advertising in the same year. ${ }^{3}$

I think there should be policies to encourage exercise of any kind: as well as helping obesity and diabetes, it is great for mental well-being. I also think there should be better education to empower people to make healthy living choices.

As well as this, there is the obvious 'elephant in the room', the measure that could prevent the greatest reduction in dental caries alone - the fluoridation of water!

Public Health England published a very informative document on the evidence base for action on sugar reduction - it is a shame to see not many of these recommendations have been implemented or acted upon yet. ${ }^{3}$

\section{References}

1. Open consultation. Advancing our health: prevention in the 2020s. 22 July 2019. Available at: https://www.gov. uk/government/consultations/advancing-our-health-prevention-in-the-2020s (accessed August 2019).

2. Hashem K M, He F J, Alderton S A, MacGregor G A Cross-sectional survey of the amount of sugar and energy in chocolate confectionery sold in the UK in 1992 and 2017. Nutrients 2019; 11: 1798.

3. Public Health England. Sugar Reduction: The evidence for action. London, October 2015. Available at: https:// assets.publishing.service.gov.uk/government/uploads/ system/uploads/attachment_data/file/470179/Sugar_ reduction_The_evidence_for_action.pdf (accessed September 2019).

4. Public Health England. Sugar reduction: Report on progress between 2015 and 2018. September 2019. Available at: https://assets.publishing.service.gov.uk/ government/uploads/system/uploads/attachment_data/ file/832182/Sugar_reduction__Yr2_progress_report.pdf (accessed September 2019). 